\title{
UTILIZING A WIEN FILTER WITHIN THE BEAM DYNAMICS SIMULATION TOOL V-CODE*
}

\author{
W. Ackermann**, W.F.O. Müller, B. Steiner, T. Weiland, \\ Technische Universität Darmstadt, Institut für Theorie Elektromagnetischer Felder (TEMF), \\ Schlossgartenstraße 8, 64289 Darmstadt, Germany, \\ J. Enders, C. Heßler, Y. Poltoratska, \\ Technische Universität Darmstadt, Institut für Kernphysik (IKP), \\ Schlossgartenstraße 9, 64289 Darmstadt, Germany
}

\section{Abstract}

Beam dynamics simulations for computationally large problems are challenging tasks. On the one hand, for the accurate simulation the electromagnetic field distribution within the whole device and the surrounding environment, it is essential to consider all necessary device components including even small geometry details, complicated material distributions and the field excitations. On the other hand, additional computational effort has to be put into precise modeling of the injected particle beam for accurate beam dynamics simulations.

Under linear conditions, it is possible to separate the field calculation of the device from the computation of the particles self-field which can result in the proper application of diverse numerical schemes for the individual field contributions. In the paper, a technique is presented by means of which the static electric and magnetic fields of a Wien filter beam line element can be treated as applied external fields within the beam dynamics simulation tool V-Code under the assumption that the interaction of the particle beam with the surrounding materials can be neglected.

\section{INTRODUCTION}

A schematic configuration of the analyzed Wien filter is shown in Fig. 1. It is installed in a test stand to set up a new polarized electron gun at the superconducting linear accelerator in Darmstadt (S-DALINAC) [1] which is designed to supplement the reliable non-polarized electron source to extend the range of experiments.

The polarized electrons are extracted from a strained gallium arsenide photo cathode which can be illuminated by laser pulses of a proper time structure and intensity. After extraction, the polarized electrons are bent into the injector beam line with the help of an alpha magnet. In order to align the polarized electrons to the experimental needs, a Wien filter beam line element acting as a spin rotator is included.

In Fig. 1, the main filter components necessary to excite

\footnotetext{
* This work was partially funded by EUROFEL (RIDS-011935), DESY Hamburg, and DFG (SFB 634).

**ackermann@temf.tu-darmstadt.de
}

05 Beam Dynamics and Electromagnetic Fields the electric and magnetic fields are visualized. To get an insight into the element, part of the housing and the magnetic yoke have been cut away. A static homogeneous magnetic flux density in horizontal direction is obtained by impressing a constant electric current through the symmetric coils where for shielding reasons additional magnetic conductive materials are inserted. The corresponding electric field is excited by a high voltage source applied to the electrodes resulting in a static vertically oriented homogeneous electric field strength.

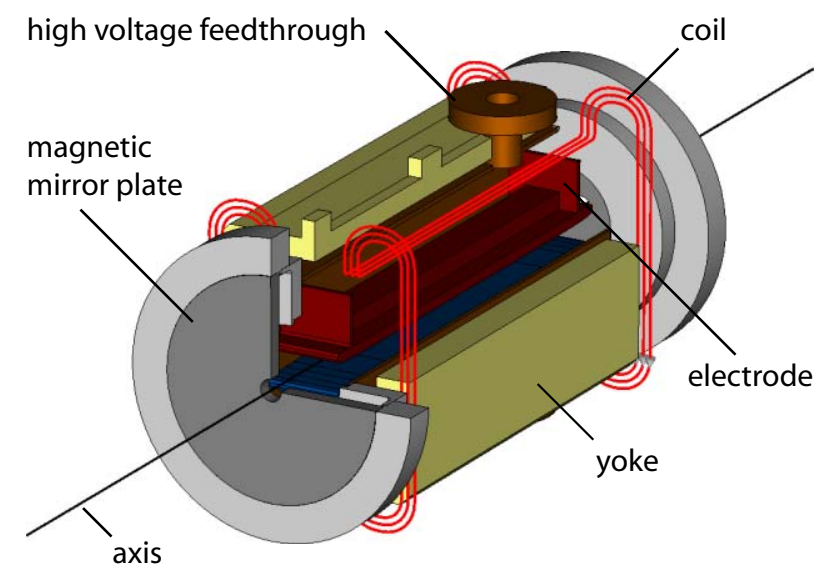

Figure 1: Schematic computational model of a Wien filter.

\section{FIELD CALCULATION}

Prior to realistic beam dynamics simulations for either practical design purposes or academic researches the threedimensional (3D) distribution of the electric and magnetic fields in the whole structure has to be determined. In contrast to the design process where all components of the fields are necessary, the subsequent beam dynamics simulations rely upon the field values only in the areas covered by the particle beam. Due to the fact that those areas are also accessible by measurement devices, the measured data can serve as reference.

During the design process when no real physical device is available, only simulated data can be used. Once a detailed simulation model has been set up, all necessary field D05 Code Developments and Simulation Techniques 
components can be utilized for further needs. All computations have been performed numerically within the CST Design Environment [2] in two steps. Firstly, a magnetostatic simulation is run to obtain the full 3D distribution of the magnetic field strength and the magnetic flux density. After that, an electrostatic simulation is initiated delivering a full 3D distribution of the electric field strength and electric flux density.

The whole Wien filter is discretized with the help of a computational mesh where all geometrical details in connection with the material properties have to be resolved. After the consecutive runs of the electrostatic and magnetostatic solvers, all needed field components are available.

\section{FIELD EXTRACTION}

Since the beam dynamics simulations require a small fraction of the full field data only this necessary information is extracted from the whole computational model. Usually, the fields in the areas accessible by the particle beam is transferred as a 3D field map. A significant reduction of data can be achieved while maintaining the quality if the field possesses certain symmetry properties.

\section{Multipole Expansion}

One design goal for the Wien filter is to obtain an ideal homogeneous transversal electric field strength and magnetic flux density mutually perpendicular to each other within the whole domain neglecting fringe field effects. In the real setup those perfect fields can hardly be realized and one has to optimize the final setup in a way that the physical needs are safely satisfied. To measure the quality of the fields, the coefficients of their multipole expansions are applied. For this purpose one monitors the tangential field components on different radii of concentric circles transversal to the axis of the Wien filter.

In Fig. 2, the multipole coefficients in logarithmic scale normalized to the dipole field strength are displayed up to order 40. From the specified data one can conclude that independent of the radius and field type, the sextupole component represents the most important disturbance to the aimed dipole fields. In this connection the magnetic flux density is more concerned than the electric field strength. Furthermore, the analysis also identifies that even for the largest examined radius of $r=6 \mathrm{~mm}$ those parasitic field components are negligible compared to the dominant dipole components of the fields.

\section{Fringe Fields}

The multipole expansion of the electric as well as the magnetic field leads to the conclusion that no further field component except the dipole part has to be included during the extraction procedure. However, the preferable instantaneous appearance of fields inside the Wien filter device is too simplistic and is not able to represent the physical behavior of charged particles passing through.
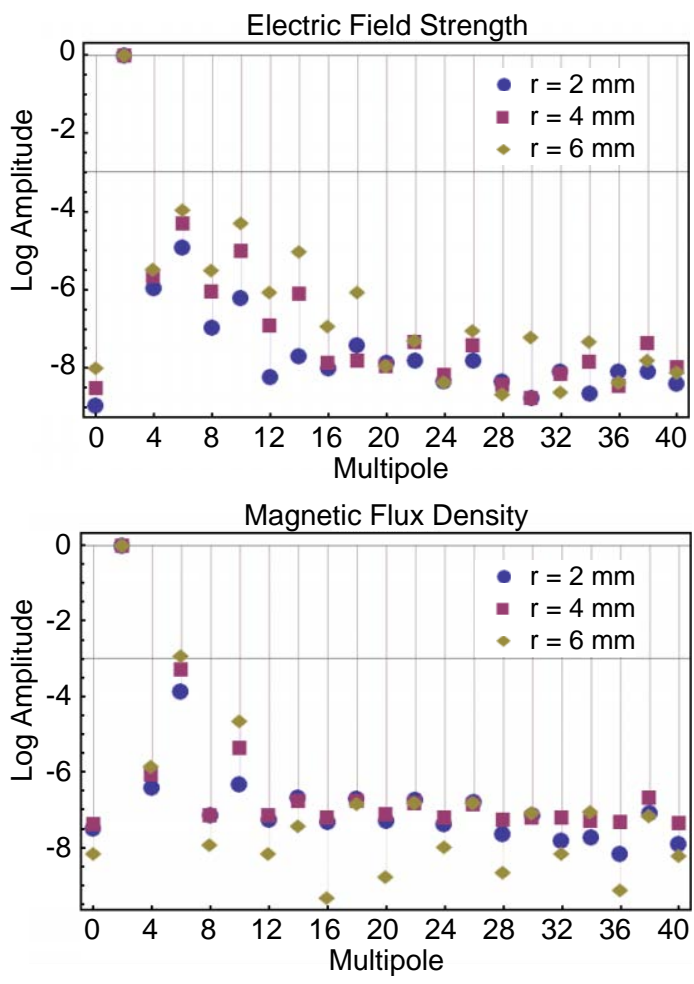

Figure 2: Normalized multipole moments of the electric field strength and the magnetic flux density in logarithmic scale measured in the center of the Wien filter.

A rigorous derivation of a proper functional description can be obtained with the help of MAXWELL equations in free space. Following the so called paraxial approach one is able to derive for example the electrical field components in cylindrical coordinates $(r, \varphi, z)$

$$
\begin{aligned}
& E_{r}=\left[\begin{array}{ll}
\alpha(z) & -\frac{3}{8} r^{2} \alpha^{\prime \prime}(z)+\ldots
\end{array}\right] \cdot \sin \varphi \\
& E_{\varphi}=\left[\alpha(z) \quad-\frac{3}{8} r^{2} \alpha^{\prime \prime}(z)+\ldots\right] \cdot \cos \varphi \\
& E_{z}=\left[\alpha^{\prime}(z) r-\frac{1}{8} r^{3} \alpha^{\prime \prime \prime}(z)+\ldots\right] \cdot \sin \varphi
\end{aligned}
$$

as a function of the longitudinal variable dipole weight $\alpha(z)$. The specified derivation is in particular valid for the vacuum region inside the beam tube. All described fields are subsequently used in beam dynamics simulations in Cartesian coordinates leading to

$$
\begin{aligned}
& E_{x}=0 \quad-\frac{1}{4} \alpha^{\prime \prime}(z) x y \quad+\ldots \\
& E_{y}=\alpha(z) \quad-\frac{1}{8} \alpha^{\prime \prime}(z)\left(x^{2}+3 y^{2}\right)+\ldots \\
& E_{z}=\alpha^{\prime}(z) y-\frac{1}{8} \alpha^{\prime \prime \prime}(z)\left(x^{2}+y^{2}\right) y+\ldots .
\end{aligned}
$$

The corresponding series expansion of the magnetic flux density can be obtained from the electrical one by exchanging both the $x$ and $y$ coordinates as well as the $x$ and $y$ components. In Fig. 3, the extracted dipole strength as a function of the longitudinal coordinate is specified. From the series expansion one can conclude that the evaluation of the main field component along the longitudinal axis $(x=y=0)$ automatically yields the appropriate dipole strength.

D05 Code Developments and Simulation Techniques 


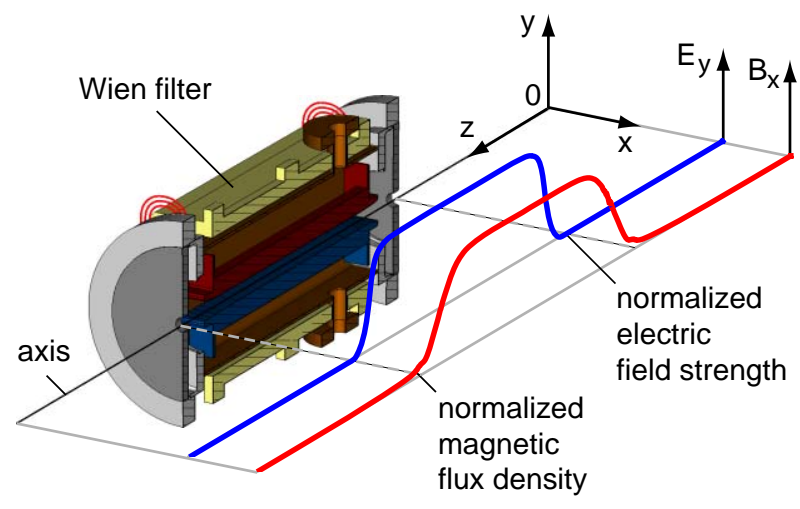

Figure 3: Distribution of the main field components along the longitudinal coordinate of the Wien filter.

\section{BEAM DYNAMICS SIMULATION}

Once the external electric field strength together with the magnetic flux density are known at least in those areas of the device which are accessible by charged particles, the desired beam dynamics studies can be performed.

\section{Computational Model}

Instead of tracking individual charged particles in given external fields, the model of ensembles for beam dynamics simulations [3] can be successfully applied if one is interested in collective parameters like mean positions and dimensions, total energy and energy spreads as well as projected emittances rather than individual particle dynamics.

Any involved ensemble is modeled with the help of the moment approach [4]. In the frame of this approach the moments of the particle distribution function are applied instead of the density function itself. The time evolution of all involved moment parameters is expressed as follows [5]:

$$
\begin{aligned}
\frac{\partial<\mu>}{c \partial t} & =<\operatorname{grad}_{<\vec{r}>}(\mu)>\cdot<\frac{\vec{p}}{\gamma}> \\
& +<\operatorname{grad}_{<\vec{p}>}(\mu)>\cdot<\frac{\vec{F}}{m_{0} c^{2}}> \\
& +<\operatorname{grad}_{\vec{r}}(\mu) \cdot \frac{\vec{p}}{\gamma}>+<\operatorname{grad}_{\vec{p}}(\mu) \cdot \frac{\vec{F}}{m_{0} c^{2}}>
\end{aligned}
$$

where the notation $\langle\mu\rangle$ is used to specify the weighted mean value of any parameter $\mu$. The weighting itself is performed via integrating over the whole phase space using the phase space density function as a weight. Usually, the parameters $\mu$ are chosen such that the first order raw moments as well as the higher order centralized moments are obtained during the weighting process.

The specified Lorentz force $\vec{F}=q \cdot(\vec{E}+\vec{v} \times \vec{B})$ can be separated into the internal space charge force $\vec{F}_{\mathrm{Sc}}$ and the external force $\vec{F}_{\text {ext }}$ stipulated by the specific setup of the single beam line elements. The knowledge of a proper series expansion for the electric field strength $\vec{E}$ and the magnetic flux density $\vec{B}$ enables to construct the necessary series description also for the applied forces. Once all se05 Beam Dynamics and Electromagnetic Fields ries terms are stated, the beam dynamics simulations based on the moment approach can be performed.

\section{Implementation}

Any manual implementation of the fundamental system of ordinary differential equations is a time-consuming task: in the lowest order approximation already 27 degrees of freedom have to be considered to construct the numerical update equations which increases further to 83, 209, 461, $923, \ldots$ if higher oder moments are considered. The internal feedback as well as the interaction with the surroundings have also to be taken into account where the influence of external forces varies from one beam line elements to another so that each contribution has to be reimplemented in any particular case.

However, it is possible to create all the involved update equations automatically with the help of symbolic algebra transformations. Following this route, the fundamental equations are generated right from the series expansion of the applied fields. The only manual work herein consists in providing a physically motivated field description. For the beam dynamics simulation tool $\mathrm{V}$-CODE [5] the proposed technique has been recently implemented. A successful application of the code can be found in [6].

\section{CONCLUSIONS}

In the paper the utilization of a Wien filter within the beam dynamics simulation tool $\mathrm{V}$-CODE is demonstrated. As the key ingredient a series expansion of the applied forces is mandatory leading to the necessity of providing a series expansion for the electric and magnetic fields. In case the fields possess certain symmetries, an extremely efficient way to transfer all field components from the field solvers to the beam dynamic simulation software can be applied. In particular, the proposed axial multipole expansion can be incorporated in a straightforward way into the moment approach. This advantage can be fully exploited within the beam dynamics simulation of a Wien filter if all contributions from higher order multipoles are negligible.

\section{REFERENCES}

[1] A. Richter, "Operational Experience at the S-DALINAC", EPAC, Sitges, 1996, p.110.

[2] CST - Computer Simulation Technology, Bad Nauheimer Str. 19, D-64289 Darmstadt, Germany, www.cst.com.

[3] A. Novokhatski, T. Weiland, "The Model of Ensembles for the Beam Dynamics Simulation”, PAC, New York, 1999.

[4] P. J. Channell, "The Moment Approach to Charged Particle Beam Dynamics", IEEE Trans. Nucl. Sci., NS-30, No. 4, Page 2607, 1983.

[5] W. Ackermann, T. Weiland, "Efficient Time Integration for Beam Dynamics Simulations Based on the Moment Method", ICAP, Chamonix Mont-Blanc, 2006.

[6] B. Steiner et al., "Wien Filter as a Spin Rotator at low Energy", these proceedings.

D05 Code Developments and Simulation Techniques 